\title{
Outcomes of colonoscopy with non-anesthesiologist- administered propofol (NAAP): an equivalence trial
}

\section{(ㄷ)(1) $\odot$}

Authors

Marco Alburquerque ${ }^{1,2}$, Antonella Smarrelli ${ }^{1}$, Julio Chevarria Montesinos ${ }^{3}$, Sergi Ortega Carreño ${ }^{4}$, Ana Zaragoza Fernandez ${ }^{4}$, Alba Vargas García ${ }^{1,2}$, Cesar Ledezma Frontado', Lluís Vidal' ${ }^{1}$, Montserrat Figa Francesch², Ferrán González-Huix Lladó ${ }^{2,5}$

Institutions

1 Department of Gastroenterology, Hospital de Palamós, Girona, Spain

2 Department of Gastroenterology, Clínica Girona, Girona, Spain

3 Department of Nephrology, Beaumont Hospital, Dublin, Ireland

4 Department of Nursing, Hospital de Palamós, Girona, Spain

5 Department of Gastroenterology, Arnau de Vilanova University Hospital, Lleida, Spain

submitted 1.11 .2020

accepted after revision 19.2.2021

Bibliography

Endosc Int Open 2021; 09: E1070-E1076

DOI 10.1055/a-1452-9242

ISSN 2364-3722

(C) 2021. The Author(s).

This is an open access article published by Thieme under the terms of the Creative Commons Attribution-NonDerivative-NonCommercial License, permitting copying and reproduction so long as the original work is given appropriate credit. Contents may not be used for commercial purposes, or adapted, remixed, transformed or built upon. (https://creativecommons.org/licenses/by-nc-nd/4.0/)

Georg Thieme Verlag KG, Rüdigerstraße 14,

70469 Stuttgart, Germany

Corresponding author

Marco Alburquerque, Hospital de Palamos -

Gastroenterology, Calle Hospital 36 Palamós Girona

Catalunya 17230, Spain

Fax: + 34972600160

nictalope7@gmail.com

\section{ABSTRACT}

Background and study aims Efficacy and safety of NAAP for gastrointestinal endoscopy have been widely documented, although there is no information about the outcomes of colonoscopy when the endoscopist supervises the sedation. In this context, the aim of this trial was to determine the equivalence of adenoma detection rate (ADR) in colorectal cancer (CRC) screening colonoscopies performed with non-anesthesiologist-administered propofol (NAAP) and performed with monitored anesthesia care (MAC).

Patients and methods This was a single-blind, non-randomized controlled equivalence trial that enrolled adults from a national CRC screening program (CRCSP). Patients were blindly assigned to undergo either colonoscopy with NAAP or MAC. The main outcome measure was the ADR in CRCSP colonoscopies performed with NAAP.

Results We included 315 patients per group. The median age was $59.76 \pm 5.81$ years; $40.5 \%$ of patients were women. The cecal intubation rate was $97 \%, 81.8 \%$ of patients had adequate bowel preparation, withdrawal time was $>6$ minutes in $98.7 \%$, and the median global exploration time was $24.25 \pm 8.86$ minutes (range, $8-70$ minutes). The ADR was $62.9 \%$ and the complication rate $(C R)$ was $0.6 \%$. Analysis by intention-to-treat showed an ADR in the NAAP group of $64.13 \%$ compared with $61.59 \%$ in the MAC group, a difference (SADR) of $2.54 \%, 95 \% \mathrm{Cl}:-0.10$ to 0.05 . Analysis by per-protocol showed an ADR in the NAAP group of $62.98 \%$, compared with $61.94 \%$ in the MAC group, $\delta A D R$ : $1.04 \%, 95 \% \mathrm{Cl}:-0.09$ to 0.07 . There was no difference in CR (NAAP: 0,63 vs. MAC: 0.63 ); $P=1.0$.

Conclusions ADR in colorectal cancer screening colonoscopies performed with NAAP was equivalent to that in those performed with MAC. Similarly, there was no difference in complication rates.

\section{Introduction}

Traditionally, there are only two methods for sedation in gastrointestinal endoscopy (GIE): standard sedation (SS) and monitored anesthesia care (MAC). In the former, intravenous drugs such as benzodiazepines and opioids are used, the target level is moderate sedation and the method is supervised by an endoscopist. In MAC, the choice of the drugs and the target level sedation are supervised by an anesthesiologist [1,2]. In recent years, in countries where non-anesthesiologist physicians 
can administer propofol, a new method has emerged: non-anesthesiologist-administered propofol (NAAP). In NAAP, propofol is administered alone or combined with other agents, the target level sedation is moderate or deep [1-3], and an endoscopist with additional training in administration of sedo-analgesic drugs and airway management supervises it. Thus, NAAP evolved from SS and is an alternative to MAC.

Since the introduction of NAAP, we have collected a lot of information about its efficacy and safety; currently, outcomes of endoscopist-directed sedation (EDS) are comparable with SS and MAC [4-9]. In agreement with this data, a more recent study including 1.38 million procedures with more than 880,000 colonoscopies and more than 508,000 gastroscopies compared risk of serious adverse events with MAC and EDS. This study concluded that the safety of the methods in colonoscopy was equivalent but that overall, MAC was riskier in gastroscopy [4]. Similar data have been reported by other authors [58]. Furthermore, EDS during colonoscopy has been described as the safest among all gastrointestinal endoscopic procedures performed with this sedation method $[4,9]$. Conversely, there is no information about the outcomes of colonoscopy itself when the endoscopist has to fulfill the additional task of supervising the sedation. This is probably due to the difficulty in measuring these outcomes because they depend on various factors, such as equipment, endoscopist training, adequate bowel preparation, and patient tolerance [10-12], among others. However, the advent of quality indicators for colonoscopy $[13,14]$ has facilitated quantification of objective parameters and monitoring and comparison of them.

The unquestionable advantages of sedation for GIE imply an additional task for the endoscopist. At least in theory, this would distract the endoscopist and could have a negative impact on colonoscopy. In this context, we have done this research to determine whether the adenoma detection rate (ADR) in colorectal cancer (CRC) screening colonoscopies performed with NAAP is equivalent to that for those performed with MAC)

\section{Patients and methods}

\section{Study design}

We conducted a single-blind, non-randomized controlled equivalence trial at a single institution from January 2017 to December 2018. Colonoscopies were performed by two expert endoscopists who had more than 7 years of experience in endoscopy and 1 year of experience in colonoscopy for CRC screening. Both had ADRs $>50 \%$ and acceptable complication rates.

The two endoscopists alternated between colonoscopy schedules with NAAP and MAC.

In NAAP, an expert nursing team directed by an endoscopist administered the sedation. This team had previously completed more than 3500 NAAP sedations for GIE. For MAC, different anesthesiologists directed the sedation, according to their usual schedules.
After signing the consent form, patients were instructed to perform bowel preparation following our usual colon cleansing protocol.

The local Research Committee and Research Ethics Committee approved our research (IRB approval code: 42319_TDA_ANE. V2.0:02/05/19). The trial then was registered in ClinicalTrials.gov. (NCT03922074). All authors reviewed and approved the final version of the manuscript.

\section{Patients}

Adults between 50 and 69 years old were recruited from the Spanish National CRC screening program (CRCSP). After a positive fecal occult blood test (FOBT), they were blindly assigned to undergo colonoscopy with either NAAP or MAC by the CRCSP office following return of the FOBT results, patient availability for colonoscopy, and the availability of slots in the endoscopy schedule. In our hospital, there are two schedules a week for these colonoscopies: NAAP on Wednesday and MAC on Friday. The CRCSP secretary did not know who administered sedation during each schedule.

Random assignment was not possible because no researcher took part in the allocation of participants. Neither the anesthesiologists nor the endoscopists who directed sedation allocated patients.

Patients with familial colorectal cancer history were excluded.

\section{Intervention}

In the NAAP arm, all patients were monitored throughout the procedure. We monitored oxygen saturation, blood pressure, rhythm, and heart rate. Pre-oxygenation began 5 minutes before the beginning of intravenous (IV) drug administration. In all colonoscopies with NAAP, a nurse exclusively administered the IV drugs and monitored the patient. At the beginning of the procedures, we administered a fentanyl infusion in bolus: $75 \mu \mathrm{g}(1.5 \mathrm{~mL})$ and an initial propofol dose $(10 \mathrm{mg} / \mathrm{mL})$ in bolus of 0.5 to $2.5 \mathrm{mg} / \mathrm{kg}$ followed by a maintenance dose of 20 to $60 \mathrm{~mL} / \mathrm{h}$ through a target-controlled infusion (TCI) pump. In this way, we achieved a moderate to deep level of sedation (Observer's Assessment of Alertness/Sedation Scale [OAAS]: 1 to 3) [15].

For patients assigned to the MAC arm, the anesthesiologist directed the choice of the IV drugs and the target level of sedation. The Anesthesiologist usually administered propofol plus other agents (e.g. benzodiazepines, opioids, ketamine) in doses he or she considered convenient.

Colonoscopies were performed per standard of care using Evis Exera III Video colonoscopes CF-HQ190 and CF-H185 L/I by Olympus. Colonoscope insertion began with the patient on the left lateral position, which was maintained until the cecum was reached. Auxiliary maneuvers (e.g. specific abdominal pressure or change of the patient position) were used as appropriate. Bowel preparation was evaluated using the Boston Bowel Preparation Scale [16].

Colonic polyps were classified according to the Paris Classification [17] and their size, estimated by comparison of one with an open standard biopsy forceps. Anatomic location and resec- 
tion techniques also were documented. Finally, a case report form was filled out for each participant immediately before and after the procedure.

Patients were followed up for 8 weeks by one researcher who reviewed their medical records and documented polyp histopathology and the appearance of any adverse events (AEs) associated with colonoscopy.

In addition, the cost of personnel for sedation was calculated by multiplying the global exploration time by 0.89 euros when the anesthesiologist administered it and 0.49 euros when the nurse did it. These prices were the average personnel cost per minute in our hospital.

\section{Outcomes}

Our main outcome measure was the ADR in CRCSP colonoscopies performed with NAAP. Secondary outcomes measures were the advanced ADR ( $A A D R)$, sessile serrated ADR (SSADR), mean number of adenomas per procedure (MAP), and the complication rate (CR) associated with these colonoscopies. We defined these indicators based on widely accepted definitions $[13,18-21]$. Regarding sessile serrated adenomas (SSAs), we considered a histological definition, i.e., a pathologist defined these lesions. In addition, SSAs $\geq 1 \mathrm{~cm}$ or SSAs with dysplasia were considered advanced adenomas $[18,19]$.

\section{Sample size and statistical analyses}

To assess the equivalence of ADR in CRC screening colonoscopies performed with NAAP and performed with MAC, we assumed an expected ADR per group $=40 \%$ [22], an equivalence margin $=10 \%$, a $95 \%$ two-sided confidence interval $(95 \% \mathrm{Cl})$, and an allocation rate $1: 1$. With these assumptions Equivalence Tests for the Difference between the two proportions were applied. Thus, 296 patients per group were calculated.

An initial descriptive analysis of the main variables was done. We estimated the measures of central tendency and statistical dispersion. Chi-squared or Fisher exact tests were used to determine whether there was a significant difference between the frequencies. The $t$-test was used to compare the quantitative variables. The analyses were performed on an intentionto-treat basis. Per-protocol analyses were used as sensitivity analyses for the main outcome. There were no protocol violations.

Finally, the equivalence was confirmed when the $95 \% \mathrm{Cl}$ for the difference between both ADRs (NAAP vs. MAC) was entirely within the equivalence range of -0.10 to +0.10 .

\section{Results}

We included 630 patients with median age $59.76 \pm 5.81$ years, $40.5 \%$ of whom were women ( $\mathbf{F i g . 1}$ ). The cecal intubation rate $(C I R)$ was $97 \%$, adequate bowel preparation (ABp) was $81.8 \%$, the requirement for a withdrawal time $>6$ minutes $\left(\mathrm{WT}_{6 \mathrm{~m}}\right)$ was met in $98.7 \%$, and the global exploration time (ET) was $24.25 \pm 8.86$ minutes (range, $8-70 \mathrm{~min}$.). The ADR was $62.9 \%$, the advanced ADR (aADR) was $37.3 \%$, the sessile serrated ADR (sSADR) was $5.2 \%$, and the mean number of adenomas per procedure (MAP) was $1.53 \pm 1.75$. The complication

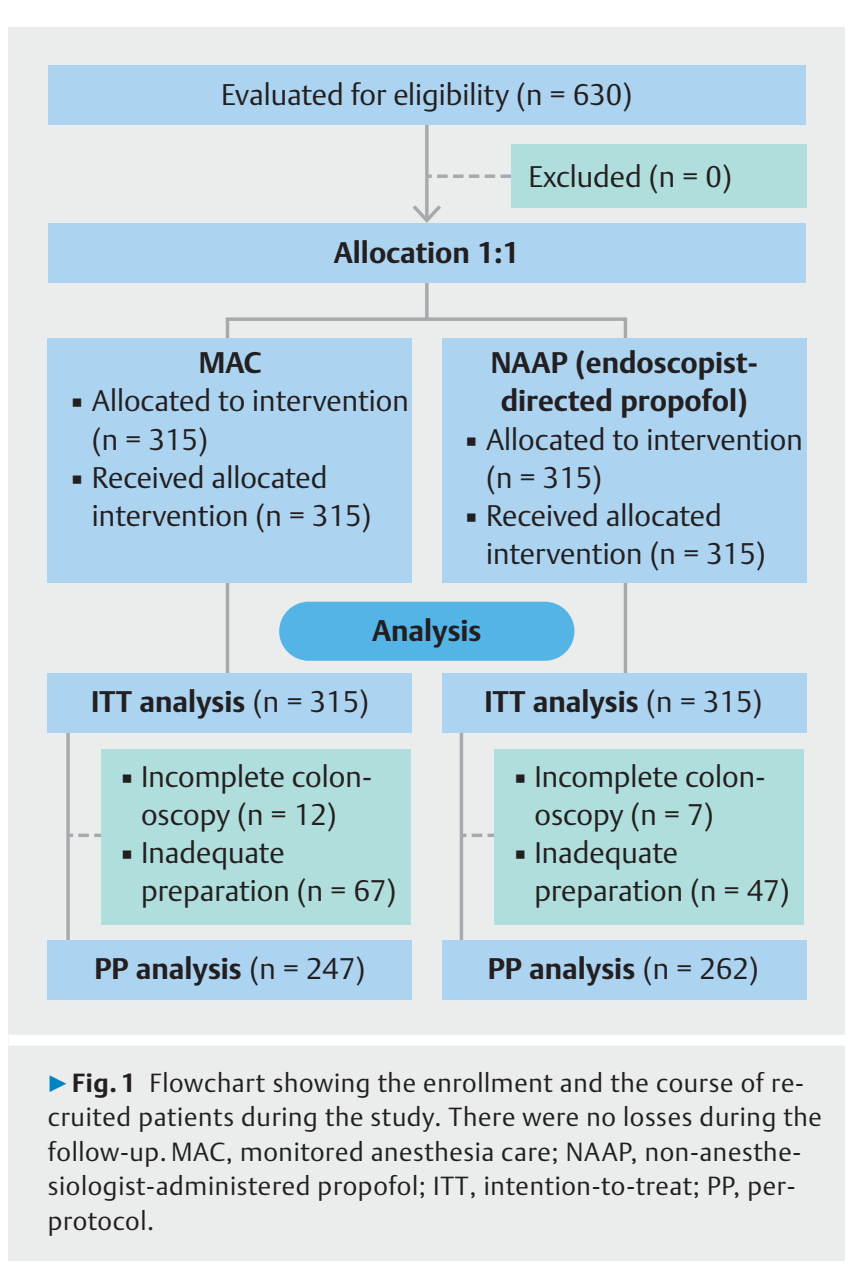

rate $(C R)$ was $0.6 \%$. All of the results except ET were comparable in the MAC and NAAP groups, although that difference disappeared when incomplete and inadequately prepared colonoscopies were excluded. The endoscopist was the only factor associated with a significant difference in ITT and PP analysis ( $>$ Table 1 and $>$ Table 2 ).

Analysis by ITT showed an ADR in colonoscopies performed with MAC of $61.59 \%$ compared with $64.13 \%$ performed with NAAP, difference ( $\delta A D R): 2.54 \%, 95 \% \mathrm{Cl}:-0.10$ to 0.05 . Analysis by PP showed an ADR in colonoscopies performed with MAC of $61.94 \%$ compared with $62.98 \%$ performed with NAAP, $\delta A D R$ : 1.04\%, $95 \% \mathrm{Cl}:-0.09$ to 0.07 . ( Table 3 and $>$ Fig. 2 ).

One post-polypectomy bleed and one splenic injury occurred in the MAC group and one post-polypectomy colonic perforation and one case of post-polypectomy syndrome occurred in the NAAP group. No differences were found between the groups in CR: MAC 0.63 vs. NAAP $0.63(P=1.0)$ ( Table 1).

Regarding the endoscopists $(E), E_{1}$ performed most of the included procedures, whereas $E_{2}$ performed $36.83 \%(n=116)$ in the MAC group and $3.49 \%(n=11)$ in the NAAP group $(P=$ $0.001)$ ( $\triangleright$ Table 1$)$. $E_{1}$ registered a superior CIR $(98.41 \%$ vs. $91.34 \%, P=0.01)$, more often met the $\mathrm{WT}_{6 \mathrm{~m}}$ requirement (99.4\% vs. $96.06 \%, P=0.01)$ and achieved a higher ADR $(64.61 \%$ vs. $55.91 \%, P=0.08)$ and MAP $(1.63 \pm 1.81$ vs. $1.15 \pm$ $1.45, P=0.01$ ) ( Table 4$)$. When the incomplete and inade- 
- Table 1 Patient characteristics and quality indicators for and outcomes of colonoscopy: ITT analysis.

\begin{tabular}{|c|c|c|c|}
\hline Sedation type & MAC $(n=315)$ & NAAP $(n=315)$ & $P$ value \\
\hline \multicolumn{4}{|l|}{ Demographic data } \\
\hline - Sex (male) (\%) & 56.83 & 62.22 & 0.168 \\
\hline - Age (year) $($ mean $\pm S D)$ & $59.37 \pm 5.73$ & $60.15 \pm 5.87$ & 0.093 \\
\hline \multicolumn{4}{|l|}{ Endoscopist } \\
\hline - Endoscopist $1(n)(\%)$ & $199(63.17)$ & $304(96.51)$ & 0.001 \\
\hline - Endoscopist 2 (n)(\%) & $116(36.83)$ & $11(3.49)$ & 0.001 \\
\hline \multicolumn{4}{|l|}{ Quality indicators for colonoscopy } \\
\hline - Cecal intubation rate (\%) & 96.19 & 97.78 & 0.244 \\
\hline " Adequate bowel preparation (\%) & 78.4 & 83.2 & 0.129 \\
\hline - Withdrawal time >6 min. (\%) & 97.78 & 99.68 & 0.069 \\
\hline - Exploration time (min.) (mean \pm SD) & $25.05 \pm 8.4$ & $23.44 \pm 9.24$ & 0.023 \\
\hline \multicolumn{4}{|l|}{ Outcomes of colonoscopy } \\
\hline - Adenoma detection rate (ADR) (\%) & 61.59 & 64.13 & 0.564 \\
\hline " Advanced ADR (\%) & 40 & 34.6 & 0.187 \\
\hline - Sessile serrated ADR (\%) & 5.41 & 5.08 & 0.860 \\
\hline " MAP (mean $\pm S D)$ & $1.42 \pm 1.64$ & $1.64 \pm 1.85$ & 0.110 \\
\hline - Complication rate (\%) & 0.63 & 0.63 & 1.000 \\
\hline
\end{tabular}

quately prepared colonoscopies were excluded, the differences between ADR (64.48\% vs. 54.08\%, $P=0.06)$ and MAP (1.62 \pm 1.83 vs. $1.12 \pm 1.39, P=0.01)$ were not only maintained but also increased, with a shorter ET $(23.63 \pm 8.07$ vs. $26.09 \pm 9.61$ min., $P=0.01)$. However, in a subanalysis taking only Endoscopist 1's results into account, there were no differences between MAC and NAAP in any of the compared measures.

The mean personnel cost per sedation during colonoscopy was significantly higher when the anesthesiologist supervised the sedation: $22.29 \pm 7.48$ vs. $11.50 \pm 4.50$ euros, $P=0.001$. Thus, MAC was more expensive than NAAP: 7022.10 vs. 3623.55 euros, respectively. Therefore, in our study, NAAP resulted in a savings of 3398.55 euros.

\section{Discussion}

Published reports to date indicate that NAAP is just as safe as MAC [4-9]. However, no information exists about NAAP's impact on the most important aspect endoscopy: procedure outcomes. Therefore, our research is the first to provide evidence in favor of use of endoscopist-directed sedation for colonoscopy.

In our study, all quality indicators for colonoscopy except ABp, which has a minimum standard of $90 \%$, were met [23]. This poor result was probably related to our colon cleansing protocol. Another we recommended a split-dose bowel preparation, patients were not advised of when to start and end it or to take the last dose as near as possible to their procedure time for colonoscopies done in the morning and for afternoon colonoscopies, to take the last dose on the same day [24]. Fortunately, those inaccuracies in the patient information have been corrected.

Regarding the outcomes of colonoscopy, our ADR largely exceeded the recommended cut-off of $50 \%$ for the CRC screening program in our region [25]. Conversely, a priori, our complication rate exceeded the accepted minimum standard of $0.5 \%$ [23]; however, it should be recognized that calculation of this rate only included our sample in the denominator instead of all colonoscopies performed by the endoscopists during the same period. In doing so, both endoscopists strictly met the requirement. Furthermore, the AEs that occurred were following complex polypectomies, for which a much higher complication rate is to be expected $[26,27]$, and in our center, that rate was not exceeded [28].

Currently the ADR is the best quality indicator for colonoscopy and the only one strongly related to interval CRC risk [13, 29]; however, it is important to recognize that the ADR is an imperfect indicator $[20,21,30]$. For this reason, some reasonable alternative parameters have been proposed: advanced ADR, sessile serrated ADR, and mean number of adenomas per procedure [20, 21, 30,31], among others. Until recently, however, no cut-off points had been established for these measures $[20,21,30]$, so we were not able to evaluate these results. In addition, a comparison of our aADR, ssADR and MAP with other 
- Table 2 Patient characteristics and quality indicators for and outcomes of colonoscopy: PP analysis.

\begin{tabular}{|c|c|c|c|}
\hline Sedation type & MAC $(n=247)$ & NAAP $(n=262)$ & $P$ value \\
\hline \multicolumn{4}{|l|}{ Demographic data } \\
\hline - Sex (male) (\%) & 53.85 & 57.63 & 0.390 \\
\hline - Age (year) $($ mean $\pm S D)$ & $59.23 \pm 5.69$ & $60.15 \pm 5.91$ & 0.075 \\
\hline \multicolumn{4}{|l|}{ Endoscopist } \\
\hline - Endoscopist 1 (n)(\%) & $158(63.97)$ & $253(96.56)$ & 0.001 \\
\hline - Endoscopist 2 (n)(\%) & $89(36.03)$ & $9(3.44)$ & 0.001 \\
\hline \multicolumn{4}{|l|}{ Quality indicators for colonoscopy } \\
\hline Withdrawal time $>6$ min. $(\%)$ & 100 & 100 & - \\
\hline Exploration time (min.) $($ mean \pm SD) & $24.83 \pm 7.75$ & $23.42 \pm 8.99$ & 0.060 \\
\hline \multicolumn{4}{|l|}{ Outcomes of colonoscopy } \\
\hline - Adenoma detection rate (ADR) (\%) & 61.94 & 62.98 & 0.810 \\
\hline - Advanced ADR (\%) & 38.87 & 33.21 & 0.196 \\
\hline - Sessile serrated ADR (\%) & 4.07 & 4.58 & 0.830 \\
\hline - $\mathrm{MAP}($ mean $\pm S D)$ & $1.40 \pm 1.58$ & $1.64 \pm 1.91$ & 0.137 \\
\hline - Complication rate (\%) & 0.81 & 0.76 & 1.000 \\
\hline
\end{tabular}

- Table 3 Equivalence of ADR between MAC and NAAP.

\begin{tabular}{|c|c|c|c|}
\hline & MAC & NAAP & 95\%CI $\delta$ ADR \\
\hline ADR & & & \\
\hline - ITT & 61.59 & 64.13 & $\mathbf{- 0 . 1 0 - 0 . 0 5}$ \\
\hline . PP & 61.94 & 62.98 & $\mathbf{- 0 . 0 9 - 0 . 0 7}$ \\
\hline
\end{tabular}

ADR, adenoma detection rate; MAC, monitored anesthesia care; NAAP, nonanesthesiologist-administered propofol; ITT, intention to treat; PP, per-protocol.

published reports would have been inaccurate given the disparities among definitions, populations, and endoscopists. For instance, SSAs have been defined based on histology in some studies or on their size and location in other ones [31-33].

The demographics in our MAC and NAAP groups were comparable. In contrast, the bowel preparation tended to be better and exploration time shorter in the NAAP arm, which indicates that colonoscopies were performed more quickly in patients with adequate preparation. Indeed, in this scenario, reaching the cecum is easier and less washing is required; therefore, a thorough and complete mucosal inspection is accomplished in a shorter time [23]. On the other hand, as discussed below, our findings would have been influenced by better performance by Endoscopist 1, who performed more procedures in the NAAP arm. When we excluded the incomplete and inadequately prepared colonoscopies, the differences between NAAP and MAC disappeared.

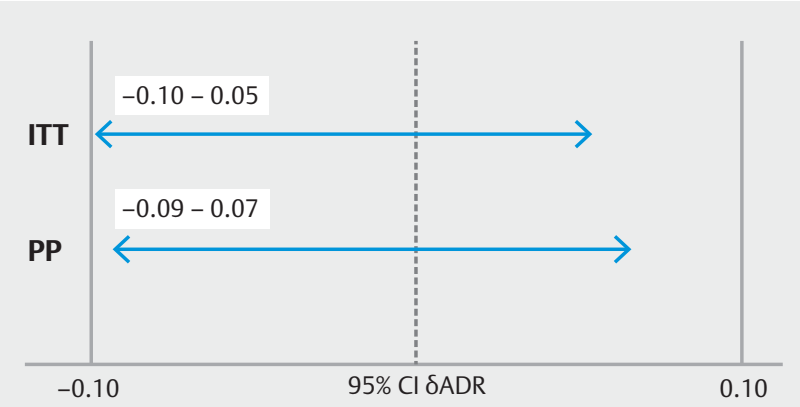

Fig. 2 95\% two-sided confidence interval for the difference between ADR (MAC vs NAAP). MAC, monitored anesthesia care; NAAP, non-anesthesiologist-administered propofol; ADR, adenoma detection rate; $\delta A D R$, difference between ADR; ITT, intentionto-treat; PP, per-protocol (analysis excluded incomplete and inadequately prepared colonoscopies).

Regarding the outcomes of colonoscopy, there were no differences between MAC and NAAP in ADR, complication rates, or other calculated measures, even when the incomplete and inadequately prepared colonoscopies were excluded.

In our study the equivalence margin, to assess the equivalence of ADR in CRC screening colonoscopies performed with NAAP and performed with MAC, was $10 \%$ so the bounds of the confidence interval were -0.10 and +0.10 . In PP analysis, the $95 \% \mathrm{Cl}$ of the difference between both ADR lay entirely within the equivalence range, Consequently, this trial has confirmed that the ADR with NAAP is equivalent to the ADR with MAC. Nevertheless, it should be noted that our results reflect 
- Table4 Quality indicators for and outcomes of colonoscopy according to endoscopist (E).

\begin{tabular}{|c|c|c|c|}
\hline & E1 $(n=503)$ & $E 2(n=127)$ & $P$ value \\
\hline \multicolumn{4}{|l|}{ Demographic data } \\
\hline - Sex (male) (\%) & 60.83 & 54.33 & 0.186 \\
\hline - Age (year) (mean $\pm S D)$ & $59.79 \pm 5.83$ & $59.65 \pm 5.73$ & 0.807 \\
\hline \multicolumn{4}{|l|}{ Quality indicators for colonoscopy } \\
\hline - Cecal intubation rate (\%) & 98.41 & 91.34 & 0.001 \\
\hline - Adequate bowel preparation (\%) & 82.83 & 77.78 & 0.197 \\
\hline - Withdrawal time> 6 min. (\%) & 99.4 & 96.06 & 0.010 \\
\hline - Exploration time (min.) $($ mean $\pm S D)$ & $23.93 \pm 8.48$ & $25.5 \pm 10.12$ & 0.109 \\
\hline \multicolumn{4}{|l|}{ Outcomes of colonoscopy } \\
\hline - Adenoma detection rate (ADR) (\%) & 64.61 & 55.91 & 0.081 \\
\hline " Advanced ADR (\%) & 36.58 & 40.16 & 0.473 \\
\hline - Sessile serrated ADR (\%) & 5.78 & 3.15 & 0.274 \\
\hline - MAP (mean $\pm S D)$ & $1.63 \pm 1.81$ & $1.15 \pm 1.45$ & 0.002 \\
\hline - Complication rate (\%) & 0.6 & 0.79 & 1.000 \\
\hline
\end{tabular}

a comparison between an anesthesiologist and an expert nursing team directed by an endoscopist in which the hypothetical additional distraction and difficulty for the endoscopist, if it existed at the beginning, had already been overcome.

Concerning the two endoscopists (E) who took part in the study, there was a clear trend toward a higher ADR in favor of $\mathrm{E}_{1}$ and a significant difference between MAP and NAAP for that endoscopist. The ADR reflects adequate inspection of the bowel mucosa [23], which in turn depends on, among other things, a complete and thorough evaluation. CIR and $\mathrm{WT}_{6 \mathrm{~m}}$, respectively, directly determine these two last requirements [23], and those were significantly higher for E1 as well. An upward trend in ADR is very important because it has been proven that, in a primary colonoscopy screening setting, a $1 \%$ increase in ADR predicted a $3 \%$ decrease in the risk of interval CRC [29]. On the other hand, although there is no accepted cut-off for MAP, we agree that in the proper circumstances, use of this indicator would be a complementary way to evaluate and compare endoscopist performance [21]. The superior results by E1 may have been associated with his training and longer experience with CRC screening colonoscopy, which has been demonstrated to improving endoscopist skills [12]. Despite the clear differences between the endoscopists and their contributions to the study, the ADRs for MAC and NAAP were probably equivalent because the sedation method did not affect endoscopist performance, as was indicated by the subanalysis of Endoscopist 1's results.

Finally, even though this trial was not an economic study, our personnel costs indicate that NAAP results in a concrete and significant savings, which would be even more significant if use of this method of sedation was expanded to other health systems.

\section{Conclusions}

In summary, from the previous data, we can conclude that ADR in colorectal cancer screening colonoscopies performed with NAAP is equivalent to ADR in colonoscopies performed with MAC. Similarly, there is no difference in complication rates.

In spite of the obvious limitations of our study, such as the absence of randomization or the fact that it was conducted at a single institution, we were able to propose an economical, effective, safer and more accessible alternative to traditional MAC without decreasing outcomes of colonoscopy. The result may be a solution to the increasing problem of lack of expert personnel in most national health systems, and specifically for supporting widespread CRC screening programs.

\section{Competing interests}

The authors declare that they have no conflict of interest.

\section{References}

[1] Vargo J], Cohen LB, Rex DK et al. Position statement: Non-anesthesiologist administration of propofol for GI endoscopy. Gastrointest Endosc 2009; 70: 1053-1059

[2] Chutkan R, Cohen J, Abedi M et al. Training guideline for use of propofol in gastrointestinal endoscopy. Gastrointest Endosc 2004; 60: $167-172$ 
[3] Waring JP, Baron TH, Hirota WK et al. Guidelines for conscious sedation and monitoring during gastrointestinal endoscopy. Gastrointest Endosc 2003; 58: 317-322

[4] Vargo JJ, Niklewski PJ, Williams JL et al. Patient safety during sedation by anesthesia professionals during routine upper endoscopy and colonoscopy: an analysis of 1,38 million procedures. Gastrointest Endosc 2017; 85: 101-108

[5] Adeyemo A, Bannazadeh M, Rigs Y et al. Does sedation type affect colonoscopy perforation rates? Dis Colon Rectum 2014; 57: 110-114

[6] Cooper GS, Kou TD, Rex DK. Complications following colonoscopy with anesthesia assistance: a population-based analysis. JAMA Intern Med 2013; 173: 551-556

[7] Agostini M, Fanti L, Gemma M et al. Adverse events during monitored anesthesia care for $\mathrm{Gl}$ endoscopy: an 8-year experience. Gastrointest Endosc 2011; 74: 266-275

[8] Wernli KJ, Brenner AT, Rutter CM et al. Risk associated with anesthesia services during colonoscopy. Gastroenterology 2016; 150: 888-894

[9] Gonzalez-Huix F, Figa M, Alburquerque M et al. Serious adverse events of nonanesthesiologist-administered propofol in relation with gastrointestinal endoscopic procedure. Gastrointest Endosc 2014; 79: 330

[10] Kaminski MF, Hassan C, Bisschops R et al. Advanced imaging for detection and differentiation of colorectal neoplasia: European society of GI endoscopy (ESGE) Guideline. Endoscopy 2014; 46: 435-449

[11] Subramanian V, Mannath J, Hawkey C] et al. High definition colonoscopy vs. standard video endoscopy for the detection of colonic polyps: a meta-analysis. Endoscopy 2011; 43: 499-505

[12] Coe SG, Crook JE, Diehl NN et al. An endoscopic quality improvement program improves detection of colorectal adenomas. Am J Gastroenterol 2013; 108: 219-226

[13] Kaminski MF, Regula J, Kraszewska E et al. Quality indicators for colonoscopy and the risk of interval cancer. N Engl J Med 2010; 362: 1795-803

[14] Von Karsa L, Patnick J et al. European Colorectal Cancer Screening Guidelines WorkingGroup. European guidelines for quality assurance in colorectal cancer screening and diagnosis: overview and introduction to the full supplement publication. Endoscopy 2013; 45: 51-59

[15] Chernik DA, Gillings D, Laine $\mathrm{H}$ et al. Validity and reliability of the Observer's Assessment of Alertness/Sedation Scale: study with intravenous midazolam. J Clin Psychopharmacol 1990; 10: 244-251

[16] Lai E, Calderwood A, Doros G et al. The Boston Bowel Preparation Scale: A valid and reliable instrument for colonoscopy-oriented research. Gastrointest Endosc 2009; 69: 620-625

[17] Paris workshop participants. The Paris endoscopic classification of superficial neoplastic lesions: esophagus, stomach and colon. Gastrointest Endosc 2003; 58: 3-43

[18] Williansom SB. Colorectal adenomas. N Engl J Med 2016; 374: 10651075
[19] Rex D, Boland R, Domiitz ] et al. Colorectal cancer screening: Recommendations for physicians and patients from the U.S. Multi-Society Task Force on Colorectal Cancer. Gastrointest Endosc 2017; 86: 1833

[20] Denis B, Sauleau EA, Gendre I et al. The mean number of adenomas per procedure should become the gold standard to measure the neoplasia yield of colonoscopy: a population-based cohort study. Dig Liver Dis 2014; 46: 176-181

[21] Wang HS, Pisegna J, Modi R et al. Adenoma detection rate is necessary but insufficient for distinguishing high versus low endoscopist performance. Gastrointest Endosc 2013; 77: 71-78

[22] Rex D, Schoenfeld P, Cohen J et al. Quality indicators for colonoscopy. Gastrointest Endosc 2015; 81: 31-53

[23] Kaminski M, Thomas-Gibson S, Bugajski M et al. Performance measures for lower gastrointestinal endoscopy: a European Society of Gastrointestinal Endoscopy (ESGE) Quality Improvement Initiative. Endoscopy 2017; 49: 378-397

[24] Hassan C, East J, Radaelli F et al. Bowel preparation for colonoscopy: European Society of Gastrointestinal Endoscopy (ESGE) GuidelineUpdate 2019. Endoscopy 2019; 51: 775-794

[25] Consell Assessor del Programa de detecció precoç de càncer de còlon i recte de Catalunya. Pla director d'oncologia. Departament de Salut. Generalitat de Catalunya. Criteris generals d'organització i funcionament del Programa de detecció precoç de càncer de còlon i recte de Catalunya. Versió 2.1-Novembre 2016. 2nd ed. Barcelona: 2016

[26] Buchner A, Guarner-Argente C, Ginsberg G. Outcomes of EMR of defiant colorectal lesions directed to an endoscopy referral center. Gastrointest Endosc 2012; 76: 255-263

[27] Ferlischt M, Moss A, Hassan C et al. Colorectal polypectomy and endoscopic mucosal resection (EMR). European Society of Gastrointestinal Endoscopy (ESGE). Clinical Guideline. Endoscopy 2017; 49: 270-297

[28] Alburquerque M, Vargas A, Sanchez I. Risk factors for incomplete endoscopic mucosal resection of large colorectal polyps Paris Is-II. Gastrointest Endosc 2018; 87: 484-485

[29] Corley DA, Jensen CD, Marks AR et al. Adenoma detection rate and risk of colorectal cancer and death. N Engl J Med 2014; 370: 12981306

[30] Bretthauer M. Detection rates during colonoscopy: What matters most? Endoscopy 2020; 52: 15-16

[31] Klair JS, Munish A, Johnson D et al. Serrated polyp detection rate and advanced adenoma detection rate from a US multicenter cohort. Endoscopy 2020; 51: 61-67

[32] Anderson J. Detection of serrated polyps: How do endoscopists rate? Endoscopy 2018; 50: 950-952

[33] Crockett S, Gourevitch R, Morris M et al. endoscopist factor that influence serrated polyp detection: a multicenter study. Endoscopy 2018; 50: 984-992 